\title{
MARÍLIA VIEIRA FEBRÔNIO
}

Citologia cérvico-vaginal inflamatória associada com atividade da doença no lúpus eritematoso sistêmico juvenil

Tese apresentada à Faculdade de Medicina da Universidade de São Paulo para obtenção do título de Doutor em Ciências

Área de Concentração: Reumatologia

Orientador: Dr. Clovis Artur Almeida da Silva

São Paulo

2006 
Dados Internacionais de Catalogação na Publicação (CIP)

Preparada pela Biblioteca da

Faculdade de Medicina da Universidade de São Paulo

Creprodução autorizada pelo autor

Febrônio, Marília Vieira

Citologia cérvico-vaginal inflamatória associada com atividade da doença no lúpus eritematoso sistêmico juvenil / Marília Vieira Febrônio. -- São Paulo, 2006.

Tese(doutorado)--Faculdade de Medicina da Universidade de São Paulo.

Departamento de Clínica Médica.

Área de concentração: Reumatologia.

Orientador: Clovis Artur Almeida da Silva.

Descritores: 1.Vagina/citologia 2.Esfregaço vaginal 3.Vaginite 4.Lupus eritematoso sistêmico 5.Adolescente 6.Displasia do colo do útero

USP/FM/SBD-404/06 
A Deus, às pacientes com lúpus eritematoso sistêmico juvenil 


\section{AGRADECIMENTOS}

À minha mãe Marisa, pelo seu exemplo de integridade, humildade e perseverança, possibilitando que eu pudesse estudar e me formar médica.

A meu irmão Rodrigo, a sua existência fez com que eu aprendesse a "dividir".

A Randy.....sua presença na minha vida fez eu não desistir....

Ao Dr.Clovis....mais do que um orientador, um grande amigo e um "pai".

Às doutoras Adriana Maluf, Lúcia Campos, Bernadete Liphaus e Paola Lotito, pelo aprendizado, amizade e convivência no serviço de Reumatologia Pediátrica do Instituto da Criança.

Aos meus grandes amigos, Ricardo e Georgiana, pela presença amiga em vários momentos dessa tese, com palavras de estímulo e carinho.

Aos amigos da Reumatologia Pediátrica: Ana Júlia, Mércia, Beth, Rosa, Daniela Lipai, Nadyesda, Aline Islabão, Adriana Jesus, Carlos, Nádia, Kátia, Renata e Aline Miranda, por compartilharmos conhecimento, ajuda e mais que tudo, amizade.

À Dra. Albertina Duarte, pela oportunidade dada em acompanhar o trabalho no ambulatório da Ginecologia do Adolescente.

Ao Grupo da Ginecologia do Adolescente da FMUSP, por me acolherem com tanto carinho durante a coleta dos dados. 
À Dra. Elsa Gay, pela ajuda na interpretação dos laudos do testes de Papanicolaou.

À Dra. Eloísa Bonfá, agradeço pelas orientações e revisão do artigo para publicação.

À Dra. Rosa Pereira pela ajuda na seleção das pacientes no ambulatório da Raumatologia.

Ao Dr.Ulisses e ao Dr.Crésio pela realização da análise estatística.

A Marisa, bibliotecária do Instituto da Criança, pela ajuda no levantamento bibliográfico.

Às funcionárias da Reumatologia, particularmente a Fátima e a Cláudia, pela colaboração. 


\section{SUMÁRIO}

\section{Resumo}

Summary

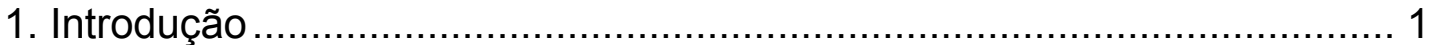

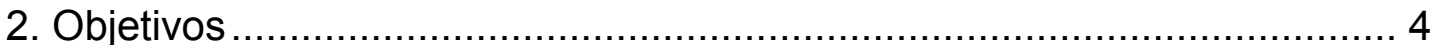

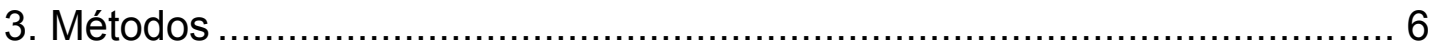

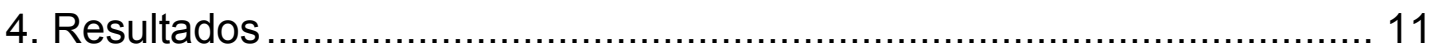

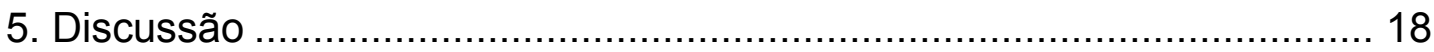

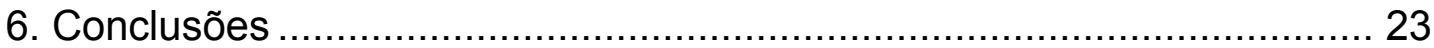

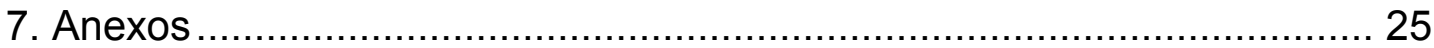

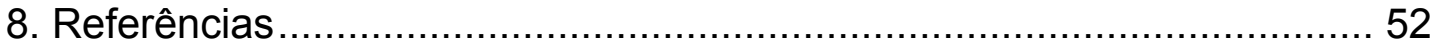




\section{RESUMO}

FEBRÔNIO MV. Citologia cérvico-vaginal inflamatória associada com atividade da doença no lúpus eritematoso sistêmico juvenil (LESJ) [tese]. São Paulo: Faculdade de Medicina, Universidade de São Paulo; 2006, 57p.

Objetivo: Avaliar a citologia cérvico-vaginal em adolescentes com lúpus eritematoso sistêmico juvenil (LESJ) e comparar com controles. Material e métodos: Cinqüenta e duas adolescentes com LESJ (critérios do American College of Rheumatology) foram comparadas com 52 controles saudáveis. Todos os esfregaços de Papanicolaou foram avaliados por uma mesma citopatologista, que desconhecia o exame ginecológico, e foram classificados de acordo com o Sistema de Bethesda, 2001. Resultados: As médias das idades das pacientes com LESJ e controles foram similares $(16,17 \pm 1,94$ versus $16,13 \pm 2,16$ anos, $p=0,92)$. A citologia cérvico-vaginal foi similar em ambos os grupos, embora as relações sexuais no último mês tenham sido menos freqüentes nas pacientes com LESJ em relação aos controles (23\% versus $59,6 \%, p=0,0003)$. Apenas uma paciente $(2 \%)$ com LESJ e duas controles (4\%) tinham displasia cervical (LIE-BG) e papilomavírus humano (HPV) $(p=1,0)$. Citologia cérvico-vaginal inflamatória foi observada em 21 (60\%) das pacientes com SLEDAI $\geq 4$ e em apenas 4 $(23 \%)$ daqueles com SLEDAI < $4(p=0,001)$. Assim como, uma maior freqüência de achados inflamatórios também foi observada em adolescentes virgens com LESJ ( $57 \%$ versus $8 \%, p=0,005)$. Vaginite por Candida spp foi observada em 7 pacientes com LESJ (14\%) e em nenhuma dos controles $(p=0,012)$, e foi associada com uso de drogas imunossupressoras $(p=0,01)$ e dose alta de prednisona $(p=0,002)$. Conclusão: Nossos achados indicam que o trato genital feminino é um órgão alvo no LESJ, pois inflamação cérvico-vaginal está associada com atividade da doença independentemente da atividade sexual. 


\section{SUMMARY}

FEBRÔNIO MV. Inflammatory cervicovaginal cytology is associated with disease activity in juvenile systemic lupus erythematosus (LESJ) [thesis]. São Paulo: Faculdade de Medicina, Universidade de São Paulo; 2006, 57p.

Objective: To evaluate cervicovaginal cytology in adolescents with juvenile systemic lupus erythematosus (JSLE) and to compare them to controls. Material and methods: Fifty-two female adolescents with JSLE (American College of Rheumatology criteria) were compared to 52 age-matched healthy controls. All Pap smears were evaluated by the same cytopathologist blinded to gynecology examination, and performed according to the Bethesda Classification System 2001. Results: The mean age of JSLE patients and controls were similar ( $16.17 \pm 1.94$ vs. $16.13 \pm 2.16$ years, $p=0.92)$. The cervicovaginal cytology was found to be similar in both groups, although sexual intercourses in the last month were less frequent in JSLE than controls $(23 \%$ vs. $59.6 \%, p=0.0003)$. Only one patient $(2 \%)$ with JSLE versus two controls (4\%) had cervical dysplasia (LGSIL) and human papilomavirus $(p=1.0)$. Inflammatory cervicovaginal cytology was observed in $21(60 \%)$ of patients with SLEDAI $\geq 4$ and only $4(23 \%)$ of those with SLEDAI $<4$ $(p=0.001)$. Likewise, a higher frequency of inflammatory changes were also observed in virgin JSLE ( $57 \%$ vs. $8 \%, p=0.005)$. Candida spp vaginitis was observed in 7 JSLE $(14 \%)$ versus none in controls $(p=0.012)$ and was associated to immunosuppressive drugs $(p=0.01)$ and high dose of prednisone $(p=0.002)$. Conclusion: Our findings supports the notion that female genital tract is a target organ in SLE since cervical inflammation is associated to disease activity independently of sexual activity. 
1. INTRODUCCÃO 
O lúpus eritematoso sistêmico juvenil (LESJ) é uma doença auto-imune multisistêmica de etiologia desconhecida acometendo especialmente mulheres jovens ${ }^{1}$. Os recentes avanços no entendimento da genética, mecanismos imunopatológicos e novas terapias do LESJ têm resultado em melhora das taxas de sobrevida de 10 anos em mais de $80 \%$ desses pacientes ${ }^{2,3}$.

Como as pacientes com LESJ apresentam uma maior sobrevida, uma preocupação com a melhora da qualidade de vida tem sido evidenciada. Neste aspecto, tornam-se adolescentes e iniciam a vida sexual com um maior risco de doenças sexualmente transmissíveis (DST), vaginite e displasia cervical.

Vaginite é uma das infecções mais freqüentes em mulheres de todas as idades $^{4}$. Além disto, evidências sugerem que vaginite, particularmente candidíase vulvovaginal, é um importante problema para pacientes com doença crônica e para aquelas que utilizam imunosupressores ${ }^{4-6}$.

O lúpus pode estar associado a um aumento do risco de displasia cervical, com freqüências variando de $24 \%$ a $36 \%$ em pacientes adultas com LES, sendo estas freqüências mais altas do que as observadas no grupo controle $^{7-11}$. Além disso, infecção pelo papilomavírus humano $(\mathrm{HPV})^{10}$ e uso de drogas imunossupressoras, particularmente pulsoterapia com ciclofosfamida endovenosa (PCE) e azatioprina, ${ }^{12-14}$ podem também ocasionar alterações no teste de Papanicolaou (Pap Test) em pacientes com LES. 
Entretanto, no lúpus juvenil ainda não existem estudos avaliando freqüência de vaginite e displasia cervical, assim como uma possível associação com uso de drogas imunossupressoras, atividade e dano cumulativo da doença. 
2. OBJETIVOS 
1. Avaliar a citologia cérvico-vaginal de adolescentes com LESJ e grupo controle.

2. Avaliar a associação entre as alterações da citologia cérvico-vaginal e a atividade da doença, o dano cumulativo e a terapêutica utilizada nas adolescentes com LESJ. 
3. MÉTODOS 


\section{Pacientes}

Cinqüenta e cinco pacientes do sexo feminino com LESJ atendidas consecutivamente, com idades entre 10 e 19 anos, e acompanhados na Unidade de Reumatologia Pediátrica e no Ambulatório da Divisão de Reumatologia do Hospital das Clínicas da Universidade de São Paulo foram selecionadas para este estudo entre janeiro de 2004 e julho de 2005 . Todas as pacientes preencheram os critérios de classificação do Colégio Americano de Reumatologia para o diagnóstico de LESJ ${ }^{15}$. O critério de inclusão foi presença de menarca e os critérios de exclusão foram a presença de gravidez no momento do estudo e diabetes mellitus. Três pacientes recusaram a participar do presente estudo. Após as exclusões, o grupo final consistiu de 52 pacientes com LESJ. O grupo controle incluiu 52 adolescentes saudáveis pareadas por idade, regularmente acompanhados na Unidade de Ginecologia do Adolescente do Hospital das Clínicas da Universidade de São Paulo. O estudo foi aprovado pela Comissão de Pesquisa e Ética do Hospital das Clínicas da Faculdade de Medicina da Universidade de São Paulo e o consentimento informado foi obtido de todos os participantes e quando necessário de seus respectivos responsáveis (Anexo 1). As classes sócioeconômicas foram avaliadas de acordo a classificação da Associação Brasileira dos Institutos de Pesquisa de Mercados (Anexo 2) ${ }^{16}$. 


\section{Avaliação clínica, laboratorial e tratamento LESJ}

A avaliação clínica foi realizada pela mesma reumatologista (MVF) e consistiu na revisão de dados sobre manifestações clínicas, exames laboratoriais e terapêuticas utilizadas. Os dados foram obtidos dos prontuários e registrados em um protocolo específico (Anexo 3). As manifestações clínicas do LES foram definidas como: envolvimento cutâneo e de mucosas (eritema malar, lúpus discóide, úlceras orais, vasculite ou fotossensibilidade), comprometimento articular (artralgia ou artrite nãoerosiva), doença neuropsiquiátrica (convulsão, psicose, depressão ou neuropatia periférica), envolvimento renal (proteinúria $\geq 0,5 \mathrm{~g} / 24 \mathrm{~h}$, presença de cilindros celulares, hematúria persistente com $\geq 10$ hemácias/campo ou insuficiência renal), doença cárdio-pulmonar (serosite, miocardite, doença pulmonar restritiva e hipertensão pulmonar) e alterações hematológicas (anemia hemolítica, leucopenia com contagem de leucócitos $<4.000 / \mathrm{mm}^{3}$, linfopenia $<1.500 / \mathrm{mm}^{3}$ e trombocitopenia com contagem de plaquetas $<$

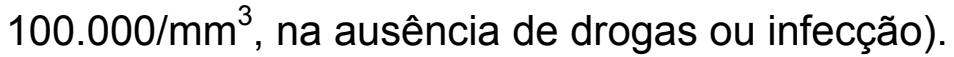

No início do estudo, foram determinados atividade de doença e dano cumulativo em todos os pacientes utilizando-se os escores: Systemic Lupus Erythematosus Disease Activity Index (SLEDAI) ${ }^{17}$ e Systemic Lupus International Collaborating Clinics/ACR - Damage Index (SLICC/ACR-DI) ${ }^{18}$. Foram determinados também dados referentes às doses atuais das drogas utilizadas no momento do estudo (prednisona, difosfato de cloroquina, metotrexate, azatioprina, PCE, ciclosporina e micofenolato mofetil) (Anexo 3). 


\section{Avaliação Ginecológica}

Um exame clínico sistemático da genitália foi realizado por uma mesma ginecologista e incluiu avaliação da vulva, hímen, vagina e colo uterino, características sexuais secundárias, de acordo com os padrões puberais de Marshall e Tanner ${ }^{19}$, e coletado o esfregaço para o teste de Papanicolaou na fase folicular do ciclo menstrual (Anexo 3).

As pacientes foram avaliadas de acordo com: idade da menarca; idade da primeira relação sexual; freqüência de relações sexuais (no último mês), número de parceiros sexuais (no último ano); vaginite (candidíase, vaginose bacteriana, Trichomonas vaginallis, HPV, etc) e contracepção (Anexo 3).

\section{Citologia cérvico-vaginal}

Os esfregaços para o Papanicolaou foram coletados nas adolescentes virgens com escova Cytobrush ${ }^{\circledR}$, que foi inserida na borda do orifício vaginal e gentilmente rodada entre $90^{\circ}$ e $180^{\circ}$, e imediatamente rolada sobre o terço externo da lâmina. Nas adolescentes sexualmente ativas, após introdução do espéculo, a citologia foi coletada com escova Cytobrush ${ }^{\circledR}$ e espátula de Ayre. O colo uterino foi visualizado e a espátula de Ayre foi inserida no orifício cervical e rodada $360^{\circ}$ com uma leve pressão, depois a escova Cytobrush $\AA$ foi inserida em dois terços do canal endocervical e rodada entre $90^{\circ}$ e $180^{\circ}$. O material da escova Cytobrush $\AA$ foi rolado sobre o terço externo da lâmina e o material da espátula de Ayre 
foi espalhado em fina camada sobre o terço médio da lâmina. Após a fixação por imersão em álcool a 95\%, os esfregaços foram imediatamente transportados ao laboratório ${ }^{20}$.

Todos os esfregaços foram avaliados por uma mesma citopatologista do nosso hospital universitário que desconhecia o exame ginecológico. Os esfregaços foram avaliados de acordo com o Sistema de Bethesda $2001 \mathrm{em}$ cinco padrões, com presença ou ausência de infecção pelo HPV: normal (alterações celulares benignas), alterações inflamatórias, células escamosas atípicas de significado indeterminado (CEASI), lesões intra-epiteliais escamosas de baixo ou alto grau (LIE-BG ou LIE-AG) e carcinoma in situ. Foram também avaliadas as identificações dos organismos (Trichomonas vaginalis, Candida spp, vaginose bacteriana, Actinomyces spp, vírus herpes simples) $)^{21}$.

\section{Análise estatística}

Os resultados foram apresentados como média \pm desvio padrão (DP) ou mediana para variáveis contínuas e percentagem para variáveis categóricas. Os dados para variáveis contínuas foram comparados através de teste "t" para avaliar diferenças entre os pacientes com LESJ e grupo controle e entre os pacientes com LESJ. Diferenças entre as variáveis categóricas foram avaliadas pelos testes do qui-quadrado ou exato de Fisher. A significância estatística foi estabelecida como $p<0,05$. 


\section{RESULTADOS}




\section{Características demográficas}

A distribuição das características demográficas revelou que as pacientes com LESJ e controles foram semelhantes com relação à idade $(16,17 \pm 1,94$ versus $16,13 \pm 2,16$ anos, $p=0,92)$, à classe sócio-econômica (classe média alta: $21 \%$ versus $21 \%$, classe média: $64 \%$ versus $52 \%$, classe média baixa: $15 \%$ versus $23 \%, p=0,33)$ e anos de educação $(9,51 \pm 1,78$ versus $9,7 \pm 1,62$ anos, $p=0,57)$.

\section{Avaliação Ginecológica}

As características sexuais secundárias de acordo com os estágios puberais de Marshall e Tanner ${ }^{19}$ foram similares em ambos os grupos, com predominância de M4P4 (48\% versus $46 \%, p=1,0)$. A média de idade da menarca nas pacientes com LESJ $(12,82 \pm 1,62$ anos, $10-17)$ foi maior em relação ao grupo controle $(11,54 \pm 1,45$ anos, $9-14, p=0,00004)$. Por sua vez, a freqüência de pacientes sexualmente ativas foi menor nas pacientes com LESJ em relação aos controles (23\% versus $60 \%, p=0,0003)$. Em contraste, nenhuma diferença foi observada com relação a: média de idade da primeira relação sexual $(15,33 \pm 1,72$ versus $14,87 \pm 1,99$ anos, $p=0,48)$, média do número de relações sexuais no último mês $(4,33 \pm 4,73$ versus $5,45 \pm 4,02$ dias, $p=0,44)$ e média do número de parceiros no último ano [1 (1-5) versus 1 
(1-10), $p=1,0]$ nas pacientes sexualmente ativas com LESJ e controles. Assim como, foram encontradas freqüências similares de contraceptivos (83\% versus $90 \%, p=0,61)$ e uso de preservativo masculino nas pacientes sexualmente ativas com LESJ e controles $(92 \%$ versus $70 \%, p=0,65)$.

\section{Citologia cérvico-vaginal}

Esfregaços normais foram observados em $50 \%$ nos dois grupos $(p=1,15)$, alterações inflamatórias em $48 \%$ das pacientes com LESJ e em $46 \%$ do grupo controle $(p=1,0)$. LIE-BG com presença de infecção pelo HPV foram observadas em apenas uma paciente com LESJ (2\%) versus duas controles $(4 \%)(p=1,0)($ Tabela 1), apesar que $27(52 \%)$ pacientes com LESJ estavam em uso de um ou mais drogas imunossupressoras (azatioprina em 17, PCE em 15, metotrexate em 11, ciclosporina em 2 e micofenolato mofetil em 1). Por outro lado, entre as 12 pacientes com LESJ e 31 controles sexualmente ativas não foram observadas diferenças em relação à freqüência de citologia normal, inflamatória e LIE-BG (25\% versus $29 \%$, $p=1,0 ; 67 \%$ versus $65 \%, p=1,0 ; 8 \%$ versus $6 \%, p=1,0$; respectivamente).

Tabela 1 - Citologia cérvico-vaginal das pacientes com LESJ e controles

\begin{tabular}{lccc}
\hline \multicolumn{1}{c}{$\begin{array}{c}\text { Citologia cérvico-vaginal, } \\
\mathbf{n}(\%)\end{array}$} & $\begin{array}{c}\text { LESJ } \\
(\mathbf{n}=\mathbf{5 2})\end{array}$ & $\begin{array}{c}\text { Controles } \\
(\mathbf{n}=\mathbf{5 2})\end{array}$ & $\mathbf{P}$ \\
\hline Citopatologia & & & \\
$\quad$ Normal & $26(50)$ & $26(50)$ & 1,15 \\
$\quad$ Inflamatória & $25(48)$ & $24(46)$ & 1,0 \\
$\quad$ LIE-BG & $1(2)$ & $2(4)$ & 1,0 \\
Organismos & & & \\
$\quad$ Vaginose bacteriana & $0(0)$ & $4(8)$ & 0,11 \\
$\quad$ Candida spp & $7(14)$ & $0(0)$ & 0,01 \\
\hline
\end{tabular}

LESJ - Lúpus Eritematoso Sistêmico Juvenil, LIE-BG - lesão intra-epitelial de baixo grau. 
$\mathrm{Na}$ citologia cérvico-vaginal, achados inflamatórios foram significativamente associados com atividade da doença nas pacientes com LESJ com e sem atividade sexual. De fato, citologia com alterações inflamatórias foi observada em $60 \%$ das pacientes com SLEDAI $\geq 4$ versus $23,5 \%$ das pacientes com SLEDAI $<4(p=0,001)$. Reforçando esse achado, apenas $37 \%$ das pacientes com SLEDAI $\geq 4$ tinham citologia normal em comparação com $77 \%$ daqueles com SLEDAI < $4(p=0,001)$ (Tabela 2).

Tabela 2 - Características da citologia cérvico-vaginal de acordo com atividade da doença

\begin{tabular}{|c|c|c|c|}
\hline $\begin{array}{c}\text { Citologia cérvico-vaginal, } \\
\text { n (\%) }\end{array}$ & $\begin{array}{c}\text { SLEDAI }<4 \\
(\mathrm{n}=17)\end{array}$ & $\begin{array}{c}\text { SLEDAI } \geq 4 \\
(n=35)\end{array}$ & $\mathbf{P}$ \\
\hline Normal & $13(77)$ & $13(37)$ & 0,001 \\
\hline Inflamatória & $4(23,5)$ & $21(60)$ & 0,001 \\
\hline LIE-BG & $0(0)$ & $1(3)$ & 1,0 \\
\hline
\end{tabular}

LESJ - Lúpus Eritematoso Sistêmico Juvenil, LIE-BG - lesão intra-epitelial de baixo grau, SLEDAI - Systemic Lupus Erythematosus Disease Activity Index.

A análise específica das pacientes virgens com LESJ revelou que achados inflamatórios foram também observados mais freqüentemente em pacientes com SLEDAI $\geq 4$ em relação as pacientes com SLEDAI $<4(40 \%$ versus $8 \%$, respectivamente, $p=0,005)$ (Tabela 3 ).

Tabela 3 - Características da citologia cérvico-vaginal em adolescentes virgens com LESJ de acordo com atividade da doença

\begin{tabular}{lccc}
\hline \multicolumn{1}{c}{$\begin{array}{c}\text { Citologia cérvico-vaginal, } \\
\mathbf{n ~ ( \% )}\end{array}$} & $\begin{array}{c}\text { SLEDAI < } \\
(\mathbf{n = 1 2 )}\end{array}$ & $\begin{array}{c}\text { SLEDAI } \geq \mathbf{4} \\
(\mathbf{n = 2 8 )}\end{array}$ & $\mathbf{p}$ \\
\hline Normal & $11(92)$ & $12(53)$ & 0,005 \\
Inflamatória & $1(8)$ & $16(57)$ & 0,005 \\
\hline LESJ - Lúpus Eritematoso Sistêmico Juvenil, SLEDAI - Systemic Lupus Erythematosus \\
Disease Activity Index.
\end{tabular}


Em contraste, nenhuma associação estatística foi observada em relação ao SLICC/ACR-DI $\geq 1$ ou $<1$ e achados inflamatórios (48\% versus $50 \%, p=1,0)$.

\section{Organismos}

Citologia com Candida spp foi evidenciada em sete (14\%) das pacientes com LESJ comparado a $0 \%$ das pacientes controles $(p=0,01)$ (Tabela 1). Todas tinham achados inflamatórios na citologia e cinco eram virgens. Assim como, nas adolescentes sexualmente ativas foi observada uma maior freqüência de Candida spp no lúpus comparada aos controles ( $17 \%$ versus $0 \%$, respectivamente), embora essa diferença não tenha sido estatisticamente significante $(p=0,07)$. Apenas duas pacientes tinham candidíase com desconforto vaginal e leucorréia e cinco apresentaram apenas leucorréia. Todas as pacientes com Candida spp foram tratadas com fluconazol oral e tópico por sete dias e apresentaram melhora dos sintomas. Estas pacientes com Candida spp estavam usando doses maiores de prednisona em relação aquelas sem essa infecção vaginal $(20,5 \pm 5$ versus 10,2 $\pm 8 \mathrm{mg}, \mathrm{p}=0,002)$. Assim como, o uso de drogas imunossupressoras (azatioprina e/ou PCE) foi maior nas pacientes com Candida spp (100\% versus $44 \%, p=0,01)$ (Tabela 4$)$. 
Tabela 4 - Dados clínicos, atividade, dano e tratamento das pacientes com LESJ com e sem infecção vaginal por Candida spp

\begin{tabular}{|c|c|c|c|}
\hline Variáveis & $\begin{array}{c}\text { Com } \\
\text { Candida } \\
\text { spp } \\
(n=7)\end{array}$ & $\begin{array}{l}\text { Sem } \\
\text { Candida } \\
\text { spp } \\
(n=45)\end{array}$ & $\mathbf{p}$ \\
\hline \multicolumn{4}{|l|}{ Características clínicas } \\
\hline Cutâneo, n (\%) & $7(100)$ & $43(96)$ & 1,0 \\
\hline Articular, n (\%) & $6(86)$ & $40(89)$ & 1,0 \\
\hline Renal, n (\%) & $7(100)$ & $37(82)$ & 0,57 \\
\hline Cardiopulmonar, n (\%) & $4(57)$ & $19(42)$ & 0,68 \\
\hline Neuropsiquiátrico, n (\%) & $2(29)$ & $10(22)$ & 0,65 \\
\hline Hematológico, n (\%) & $6(86)$ & $42(93)$ & 0,44 \\
\hline \multicolumn{4}{|l|}{ Atividade e dano do LESJ } \\
\hline SLEDAI, média \pm DP & $5,4 \pm 4,2$ & $5,5 \pm 4,4$ & 0,95 \\
\hline SLICC/ACR-DI $\geq 1, \mathrm{n}(\%)$ & $3(4)$ & $7(16)$ & 0,3 \\
\hline \multicolumn{4}{|l|}{ Tratamento } \\
\hline Dose atual de Pd (mg), média \pm DP & $20,5 \pm 5$ & $10,2 \pm 8$ & 0,002 \\
\hline Uso atual de IS, n (\%) & $7(100)$ & $20(44)$ & 0,01 \\
\hline
\end{tabular}

Além disso, as freqüências das características clínicas observadas nas pacientes com e sem Candida spp foram: envolvimento articular (86\% versus $89 \%, p=1,0)$, anormalidades hematológicas ( $86 \%$ versus $93 \%$, $p=0,44)$, comprometimento cutâneo e mucoso $(100 \%$ versus $96 \%, p=1,0)$, renal $(100 \%$ versus $82 \%, p=0,57)$, cárdio-pulmonar $(57 \%$ versus $42 \%$, $p=0,68)$ e neuropsiquiátrico $(29 \%$ versus $22 \%, p=0,65)$. A média do escore de SLEDAI $(5,4 \pm 4,2$ versus $5,5 \pm 4,4, p=0,95)$ e a percentagem do escore 
de SLICC-ACR/DI $\geq 1(43 \%$ versus $16 \%, p=0,3)$ foram comparáveis nas pacientes com LESJ com presença ou ausência de Candida spp na citologia cérvico-vaginal (Tabela 4).

Condiloma acuminado por infecção pelo HPV foi identificado na vulva, vagina e/ou colo pela colposcopia em duas controles sexualmente ativas $(6,4 \%)$ e em nenhuma paciente com LESJ $(p=1,0)$. Estas pacientes tinham LIE-BG e foram tratadas com excisão eletrocirúrgica por alça.

Vaginoses bacterianas por Gardnerella vaginallis foram observadas em três $(10 \%)$ das pacientes controles sexualmente ativas e em nenhuma das pacientes com LESJ sexualmente ativas $(p=0,54)$. Estas pacientes apresentaram uma secreção vaginal fétida que era tipicamente esparsa com uma cor acinzentada. $\mathrm{O}$ tratamento da vaginose bacteriana foi realizado com metronidazol tópico.

Trichomonas vaginalis, Actinomyces e alterações celulares compatíveis com vírus herpes simples não foram encontradas nos esfregaços das pacientes com LESJ e controles. 
5. DISCUSSÃo 
Este é o primeiro estudo que especialmente estudou a citologia cérvico-vaginal em adolescentes com LESJ e claramente demonstrou uma alta freqüência de achados inflamatórios e infecção fúngica no teste de Papanicolaou.

Vaginite é um dos maiores problemas em pacientes imunocomprometidas, tais como: diabetes mellitus, HIV e doenças autoimunes $^{6}$. De fato, situações patológicas em que o $\mathrm{pH}$ vaginal aumenta, pode resultar em supressão do crescimento dos lactobacilos e um hipercrescimento de bactérias ou fungos potencialmente patogênicos, determinando vaginite ${ }^{4}$

Em mulheres saudáveis, as infecções vaginais mais comuns são: Candida spp, vaginose bacteriana e Trichomonas vaginalis ${ }^{4}$. Por sua vez, nenhum relato tem sido descrito no lúpus em relação à vaginite, apesar de vários estudos demonstrarem que infecção é a maior causa de morbidade e mortalidade nesses pacientes ${ }^{22}$.

No presente estudo, Candida spp foi identificada como o principal microorganismo patogênico de vaginite no LESJ. A excessiva adesão epitelial e a colonização da Candida spp estão relacionadas à capacidade de aderência do fungo, assim como à redução da imunidade inata no hospedeiro comprometido. Além disto, candidíase vaginal é causada por um 
defeito ou disfunção dos linfócitos CD4+ T helper tipo 1. Além disso, os linfócitos do sangue periférico das pacientes que estão recebendo altas doses de prednisona estão diminuídos in vivo quando há estimulação antigênica pela Candida $s p p^{23}$. Neste aspecto, as pacientes com LESJ que utilizam drogas imunossupressoras devem ser consideradas candidatas às infecções fúngicas. De fato, todas as pacientes com essa infecção oportunista estavam utilizando terapias com imunossupressor e com altas doses de prednisona.

O trato genital feminino deve ser considerado como um local primário de candidíase, com probabilidade de disseminação sistêmica, pois estudos prévios demonstram que Candida albicans foi o mais freqüente microorganismo oportunista identificado em infecção fatal em adultos com LES ${ }^{24}$.

No presente estudo, não foi realizada cultura para identificar as espécies de fungos, pois a literatura reforça que habitualmente não é necessário, já que a Candida spp é sensível ao fluconazol, ${ }^{25}$ clotrimazol ou miconazol $^{4}$ tópicos, como também foi observado neste estudo.

Displasia cervical, particularmente lesões intra-epiteliais de baixo grau pelo Sistema Bethesda, foi raramente observada em pacientes com LESJ e controles. Esse achado é consistente com outros estudos em adolescentes saudáveis que evidenciaram displasia cervical em 1,9\% a $37 \%$ e LIE-BG em $3 \%$ a $24,5 \%{ }^{26-30}$. A explicação mais provável para esse achado é a reduzida freqüência de atividade sexual nas adolescentes com lúpus comparadas com as controles, possivelmente relacionada ao atraso da menarca também observado nas pacientes com LESJ. De fato, foi 
evidenciado previamente que adolescentes com LESJ apresentaram um atraso na menarca (13,5 $\pm 1,4$ anos) em comparação com 2578 adolescentes normais Brasileiras (12,5 $\pm 1,3$ anos $)^{31}$.

Por sua vez, estudos prévios em mulheres adultas com LES demonstraram uma alta prevalência de atipia cervical com LIE-BG variando de $12 \%$ a $36 \%^{8-10,12}$ e foi associada com uso de drogas imunossupressoras, particularmente PCE e azatioprina ${ }^{8,12-14}$. A baixa freqüência de displasia cervical em nossas pacientes, apesar da alta freqüência do uso de imunossupressor, sugere que a atividade sexual é provavelmente o principal fator contribuinte para as alterações no teste de Papanicolaou.

Atualmente, a incidência de anormalidades cervicais entre adolescentes está relacionada primariamente às altas taxas de atividade sexual, promiscuidade e infecção pelo HPV ${ }^{28,32-34}$. Uma possível explicação para essa associação é a maior vulnerabilidade do colo uterino "imaturo", que é coberto predominantemente por um epitélio colunar e metaplásico, com intensa replicação e diferenciação, e esse tipo de epitélio é um hospedeiro ideal para a replicação do $\mathrm{HPV}^{33}$. O uso freqüente de preservativo masculino (camisinha) pelos parceiros das nossas pacientes com LESJ e controles no presente estudo, provavelmente, contribuiu para a baixa incidência de displasia cervical e infecção vulvovaginal pelo HPV, como demonstrado recentemente em mulheres jovens sexualmente ativas ${ }^{35}$.

Por outro lado, o próprio lúpus pode predispor à inflamação cérvicovaginal, pois esta alteração na citologia foi associada a escores de SLEDAI mais elevados. Este achado também foi evidenciado em pacientes virgens 
com LESJ, que não estiveram expostas à infecção genital transmitida sexualmente, reforçando o papel da atividade da doença nas alterações inflamatórias cérvico-vaginais. Além disto, como a inflamação não foi secundária à infecção local, na maioria das pacientes estudadas neste estudo, o trato genital é possivelmente um órgão alvo no lúpus. 
6. CONCLUSÕES 
1. Displasia cervical e infecção por HPV foram raramente observadas em adolescentes com LESJ e controles. Por sua vez, candidíase vaginal foi evidenciada exclusivamente em pacientes com LESJ. Candidíase vaginal nas adolescentes com lúpus foi associada com medicamentos imunossupressores.

2. Padrão inflamatório na citologia cérvico-vaginal foi associado com atividade da doença nas pacientes com LESJ com presença e ausência de atividade sexual. 
7. ANEXOS 


\section{ANEXO 1}

\section{HOSPITAL DAS CLÍNICAS}

DA

FACULDADE DE MEDICINA DA UNIVERSIDADE DE SÃO PAULO

TERMO DE CONSENTIMENTO LIVRE E ESCLARECIDO

(Instruções para preenchimento no verso)

\section{I - DADOS DE IDENTIFICAÇÃO DO SUJEITO DA PESQUISA OU RESPONSÁVEL LEGAL}

1. NOME DO PACIENTE :

DOCUMENTO DE IDENTIDADE N ${ }^{\circ}$ : SEXO : $M \quad F$

DATA NASCIMENTO: .......................

ENDEREÇO

BAIRRO:

CEP:.

EFONE: DDD CIDADE

APTO:

2.RESPONSÁVEL LEGAL

NATUREZA (grau de parentesco, tutor, curado etc.).....

DOCUMENTO DE IDENTIDADE :

SEXO: $M$

$\mathrm{F}$

DATA NASCIMENTO.: ....................

ENDEREÇO:

.N ${ }^{\circ}$......APTO:......BAIRRO:

CIDADE:

.CEP TELEFONE:DDD (

\section{II - DADOS SOBRE A PESQUISA CIENTÍFICA}

1. TÍTULO DO PROTOCOLO DE PESQUISA : Citologia cérvico-vaginal em adolescentes do sexo feminino com Lúpus Eritematoso Sistêmico Juvenil

2. PESQUISADOR: Clovis Artur Almeida da Silva

CARGO/FUNÇÃO: Médico Responsável - Unidade de Reumatologia INSCRIÇÃO CONSELHO REGIONAL N ${ }^{\circ} \quad 64724$

UNIDADE DO HCFMUSP: Unidade de Reumatologia Pediátrica do ICr-HC-FMUSP

3. AVALIAÇÃO DO RISCO DA PESQUISA:

SEM RISCO $\quad \boldsymbol{X}$ RISCO MÍNIMO RISCO MÉDIO

RISCO BAIXO RISCO MAIOR

(probabilidade de que o indivíduo sofra algum dano como consequência imediata ou tardia do estudo)

4. DURAÇÃO DA PESQUISA : dois anos 


\section{III - REGISTRO DAS EXPLICAÇÕES DO PESQUISADOR AO PACIENTE OU SEU REPRESENTANTE LEGAL SOBRE A PESQUISA, CONSIGNANDO:}

1. justificativa e os objetivos da pesquisa: Atualmente, com a melhora do prognóstico do LESJ, os reumatologistas pediátricos encontram-se tendo que lidar com questões próprias da adolescência vividas por seus pacientes, entre elas 0 início da sexualidade e necessidade da visita com ginecologista. A citologia cérvico-vaginal ou citologia oncótica (teste de Papanicolau) é coletada rotineiramente no exame ginecológico de adolescentes, mesmo sem o início da atividade sexual, com a finalidade de identificar alterações iniciais de infecções e tumores. Alguns estudos evidenciaram aumento do risco de alterações do teste de Papanicolau em mulheres adultas com lúpus eritematoso sistêmico. A ausência de estudos na faixa etária pediátrica estimulou a realização deste projeto.

2. procedimentos que serão utilizados e propósitos, incluindo a identificação dos procedimentos que são experimentais: Os pacientes serão examinados por ginecologistas especializados em adolescentes e será coletado o teste de Papanicolaou na borda vaginal pelo ginecologista, com escova especial, mesmo em pacientes sem início da atividade sexual. Nos pacientes com atividade sexual será coletado no colo do útero.

3. desconfortos e riscos esperados: A coleta do teste de Papanicolaou na borda vaginal pelo ginecologista, com escova especial, poderá causar um leve desconforto, com dor local. Raramente leva sangramento no local e não ocasiona ruptura do hímen (perda da virgindade).

4. benefícios que poderão ser obtidos: Avaliação completa do ginecologista e identificação de possíveis infecções e tumores vaginais.

5. procedimentos alternativos que possam ser vantajosos para o indivíduo:? Seguimento e tratamento ginecológico nos pacientes que apresentarem alterações.

\section{IV - ESCLARECIMENTOS DADOS PELO PESQUISADOR SOBRE GARANTIAS DO SUJEITO DA PESQUISA: ?}

1. acesso, a qualquer tempo, às informações sobre procedimentos, riscos e benefícios relacionados à pesquisa, inclusive para dirimir eventuais dúvidas. $O$ paciente terá acesso a informação e discussão do procedimento do estudo (avaliação do ginecologista e teste de Papanicolaou).

2. liberdade de retirar seu consentimento a qualquer momento e de deixar de participar do estudo, sem que isto traga prejuízo à continuidade da assistência. 0 paciente poderá retirar seu consentimento a qualquer tempo, sem prejuízo do seu seguimento no nosso Serviço.

3. salvaguarda da confidencialidade, sigilo e privacidade. $\mathbf{O}$ estudo é sigiloso e privativo. 
4. disponibilidade de assistência no HCFMUSP, por eventuais danos à saúde, decorrentes da pesquisa. Em casos de eventuais danos à saúde relacionados a pesquisa, o paciente terá acesso ao Ambulatório de Ginecologia Infanto-Puberal do HC-FMUSP.

5. viabilidade de indenização por eventuais danos à saúde decorrentes da pesquisa. Nos casos de eventuais danos à saúde relacionados a pesquisa, o paciente terá acesso ao tratamento no Ambulatório de Ginecologia Infanto-Puberal e Unidade de Reumatologia Pediátrica do ICr do HC-FMUSP.

\section{INFORMAÇÕES DE NOMES, ENDEREÇOS E TELEFONES DOS RESPONSÁVEIS PELO ACOMPANHAMENTO DA PESQUISA, PARA CONTATO EM CASO DE INTERCORRÊNCIAS CLÍNICAS E REAÇÕES ADVERSAS.}

O estudo é retrospectivo, com análise dos prontuários.

\section{OBSERVAÇÕES COMPLEMENTARES:}

\section{VII - CONSENTIMENTO PÓS-ESCLARECIDO}

Declaro que, após convenientemente esclarecido pelo pesquisador e ter entendido o que me foi explicado, consinto em participar do presente Protocolo de Pesquisa

São Paulo, 17 de Outubro de 2003. 


\section{ANEXO 2}

\section{CLASSE SÓCIO-ECONÔMICA ABA-ABIPEMI}

(ALMEIDA, WICKERHAUSER, 1991).

A. Quem é o chefe da família na sua casa? ( ) o próprio entrevistado ( ) outros

B. Qual foi o último ano da escola que o chefe da família cursou?

\begin{tabular}{|l|c|}
\hline Grau de instrução máximo & Pontos \\
\hline Não estudou ou primário incompleto & 0 \\
\hline Primário completo ou ginásio incompleto & 05 \\
\hline Ginásio completo ou colegial incompleto & 10 \\
\hline Colegial incompleto ou universitário incompleto & 15 \\
\hline Universitário completo & 21 \\
\hline
\end{tabular}

C. Na sua casa tem?

Aparelho de vídeo cassete ( ) não ( ) sim (10 pontos)

Máquina de lavar roupas ( ) não ( ) sim (08 pontos)

Geladeira ( ) não ( ) sim (07 pontos)

Aspirador de pó ( ) não ( ) sim (06 pontos)

D. Quantos (cada item abaixo) existem em sua casa?

\begin{tabular}{|c|c|c|c|c|c|c|c|}
\hline Item & $\begin{array}{c}\text { Nenhum } \\
\text { item }\end{array}$ & 1 & 2 & 3 & 4 & 5 & $\begin{array}{c}6 \text { ou mais } \\
\text { itens }\end{array}$ \\
\hline Carros & 0 ponto & 4 pontos & 9 pontos & 13 pontos & 18 pontos & 22 pontos & 26 pontos \\
\hline $\begin{array}{c}\text { TV em } \\
\text { cores }\end{array}$ & 0 ponto & 4 pontos & 7 pontos & 11 pontos & 14 pontos & 18 pontos & 22 pontos \\
\hline Banheiros & 0 ponto & 2 pontos & 5 pontos & 7 pontos & 10 pontos & 12 pontos & 15 pontos \\
\hline $\begin{array}{c}\text { Empregada } \\
\text { mensalista }\end{array}$ & 0 ponto & 5 pontos & 11 pontos & 16 pontos & 21 pontos & 26 pontos & 32 pontos \\
\hline Rádio & 0 ponto & 2 pontos & 3 pontos & 5 pontos & 6 pontos & 8 pontos & 9 pontos \\
\hline
\end{tabular}

\begin{tabular}{|c|c|}
\hline Classes sócio - econômicas ABA-ABIPEMI & Total de pontos \\
\hline A & 89 ou mais \\
\hline B & 59 a 88 \\
\hline C & 35 a 58 \\
\hline D & 20 a 34 \\
\hline E & 0 a 19 \\
\hline
\end{tabular}




\section{ANEXO 3}

\section{PROTOCOLO DE CITOLOGIA CÉRVICO-VAGINAL EM ADOLESCENTES COM LESJ.}

\section{A- IDENTIFICACCÃO}

Nome:

Pai:

Registro:

Data de nascimento Idade atual (meses)

Data da $1^{\text {a }}$ consulta:

Raça: Sexo: Idade de início do LES (meses): Tempo de duração do LES:

Naturalidade: Data avaliação:

Endereço:

CEP: Bairro
Bairro Procedência atual:

Classe sócio-econômica da ABIPEME

Escolaridade: $T$ elefone:

\section{B- ANTECEDENTES}

Doenças familiares:

Doenças anteriores:

Vacinação:

Transfusão sangüínea:

Uso de cafeína: ( ) Sim ( ) Não (quantidade/tempo):

Uso de cigarro: ( ) Sim ( ) Não (quantidade/tempo):

Uso de álcool: ( ) Sim ( ) Não (quantidade/tempo):

Uso de drogas: （） Sim （） Não (quantidade/tempo):

C - MANIFESTAÇÕES CLÍNICAS DO LES

1. Sintomas constitucionais

1.a Febre

1.b Perda de peso

Sistema retículo-endotelial

2.a Adenomegalia

2.b Hepatomegalia

2.c Esplenomegalia

3. Cutâneo-mucoso

3.a Eritema malar

3.b Vasculite cutânea

3.c Fotossensibilidade

3.d Livedo reticular

3.e Úlceras orais

4. Músculo-esquelético

4.a Artrite

4.b Miosite

4.c Osteonecrose

5. Cardíaco

5.a Pericardite

5.b Miocardite

5.c Valvulite

6. Pulmonar

6.a Pleurite

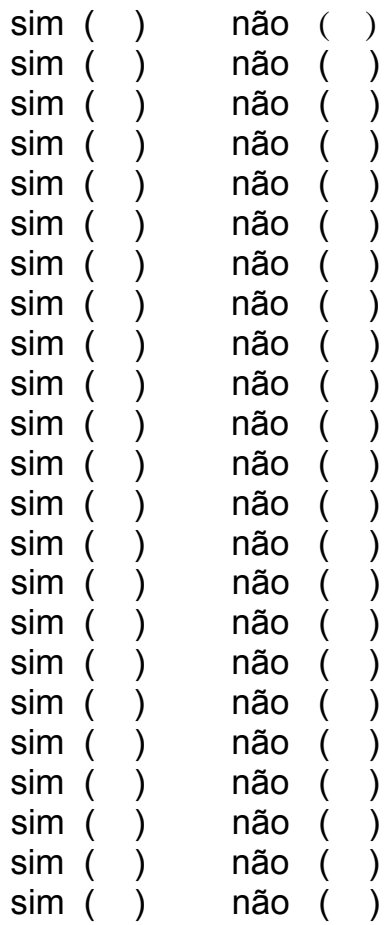


6.b Intersticial

Sistema nervoso central

7.a.Cefaléia importante

7.b Convulsão

7.c Distúrbio do comportamento

7.e Psicose

7.f Coréia

7.g Amaurose fugaz

7.h Coma

7.i Mielite transversa

8. Rim

8. 1 Hipertensão arterial 7.d Acidente vascular cerebral

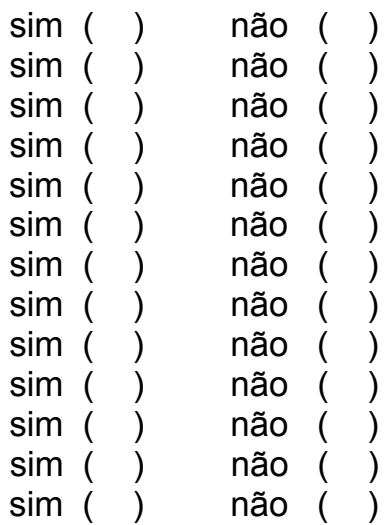

Peso e altura (curva de Marcondes - classe IV Santo André)

D - MANIFESTAÇÕES LABORATORIAIS DO LES

1. Renal

1.a Hematúria

1.b Leucocitúria

1.c Cilindrúria

1.d Proteinúria

1.e Insuficiência renal

2.f Biópsia Classe histológica OMS

2. Hematológico

2.a Anemia hemolítica

3.b Leucopenia

3.c Linfopenia

3.d Plaquetopenia

3.e Alterações de coagulação

3.f $\downarrow$ C3, $\downarrow$ C4, $\downarrow$ CH50

4. Autoanticorpos

4.a FAN

4.b anti-DNA

4.c anti-Sm

4.d anti-RNP

4.e anti-RO

4.f anti-La

4.g anti $P$

4.h anti Clp IgM

4.i anti Clp IgG

4.j anticoagulante lúpico $\operatorname{sim}(\quad)$ não ( )

$\operatorname{sim}(\quad)$ não ( )

$\operatorname{sim}(\quad)$ não ( )

$\operatorname{sim}(\quad)$ não ( )

$\operatorname{sim}(\quad)$ não ( )

$\operatorname{sim}(\quad)$ não ( )

I ( ) Ila ( ) IIb ( ) III ( ) IV ( ) V( ) VI ( )

$\operatorname{sim}(\quad)$ não $($ )

$\operatorname{sim}(\quad)$ não ( )

$\operatorname{sim}(\quad)$ não ( )

$\operatorname{sim}(\quad)$ não ( )

$\operatorname{sim}($ ) não ( )

$\operatorname{sim}(\quad)$ não ( )

$\operatorname{sim}(\quad)$ não ( )

$\operatorname{sim}($ ) não ( )

$\operatorname{sim}(\quad)$ não ( )

$\operatorname{sim}(\quad)$ não ( )

$\operatorname{sim}($ ) não ( )

$\operatorname{sim}($ ) não ( )

$\operatorname{sim}(\quad)$ não ( )

$\operatorname{sim}($ ) não ( )

$\operatorname{sim}(\quad)$ não ( )

$\operatorname{sim}(\quad)$ não ( )

$\operatorname{sim}($ ) não ( )

$\operatorname{sim}($ ) não ( )

\section{E. ATIVIDADE, CURSO E GRAVIDADE:}

- SLEDAI atual

- Curso do LES: policíclico-contínuo ( ) e remissivo ( )

- Gravidade do LES: leve ( ) e moderado-grave ( )

\section{F. TRATAMENTO UTILIZADO}

- corticosteróides (prednisona/metilprednisolona/hidrocortisona)

dose total tempo de uso

efeitos colaterais 
- pulsoterapia com ciclofosfamida

$n^{\circ}$ pulsos (dose/indução)

$\mathrm{n}^{\circ}$ pulsos (dose/manutenção)

dose total

indicação

tempo de uso

efeitos colaterais

- metotrexate

dose semanal:

dose total:

via de administração (VO,IM,SC):

efeitos colaterais:

- azatioprina

dose diária:

tempo de uso:

dose total:

efeitos colaterais:

- ciclosporina

dose diária:

tempo de uso:

dose total:

efeitos colaterais:

- $\quad$ micofenolato mofetil

dose diária:

tempo de uso:

dose total:

efeitos colaterais:

- cloroquina/ hidroxicloroquina

dose diária:

tempo de uso:

dose total:

efeitos colaterais:

- Outras drogas:

doses diária:

tempo de uso:

efeitos colaterais:

G- HISTÓRIA GINECOLÓGICA:

Menarca

Ciclos menstruais (intervalo/quantidade)

Ciclos menstruais (duração)

Dismenorréia (freqüência/ intensidade/ duração)

Infecções genitais prévias

Atividade sexual (primeira vez/ freqüência/ no de parceiros)

Masturbação (freqüência )

Presença de lubrificação vaginal

Presença de libido/orgasmo

Contracepção ( tipo de método)

EXAME FÍSICO GINECOLÓGICO:

Tanner

Grandes e pequenos lábios

Clitóris

Hímen

Vagina

Colo

Muco cervical

Toque vaginal

RESULTADO DO TESTE DE PAPANICOLAOU: 


\section{ANEXO 4}

\section{TRABALHO ENVIADO PARA PUBLICACÃO NA REVISTA LUPUS}

\section{INFLAMMATORY CERVICOVAGINAL CYTOLOGY IS ASSOCIATED WITH DISEASE ACTIVITY IN JUVENILE SYSTEMIC LUPUS ERYTHEMATOSUS}

Marilia V Febrônio ${ }^{1}$, Rosa M R Perreiral ${ }^{1}$, Eloísa Bonfá ${ }^{1}$, Albertina D Takiuti ${ }^{2}$, Elsa A.G. Pereyra², Clovis A. A. Silva ${ }^{3}$.

From Rheumatology Division ${ }^{1}$, Gynecology Division², Pediatric Rheumatology Unit of the Department of Pediatrics ${ }^{3}$, University of São Paulo, São Paulo, Brazil.

This study was supported by Conselho Nacional de Desenvolvimento Científico e Tecnológico - CNPQ (grants 304756/2003-2 to EB and $302469 / 2005-2$ to CAAS).

Address reprint requests to:

Marilia V Febrônio

Disciplina de Reumatologia, Faculdade de Medicina da Universidade de São Paulo

Av. Dr. Arnaldo 455, $3^{\circ}$ andar, São Paulo - SP - Brazil, CEP - 01246-903

FAX: $0055113061-7490$

E-mail-reumato@usp.br

Running Title: Cervicovaginal cytology in JSLE 


\section{SUMMARY}

To evaluate cervicovaginal cytology in adolescents with juvenile systemic lupus erythematosus (JSLE) and to compare them to controls. Fifty-two female adolescents with JSLE (ACR criteria) were compared to 52 agematched healthy controls. All Pap smears were evaluated by the same cytopathologist blinded to gynecology examination (Bethesda 2001). The

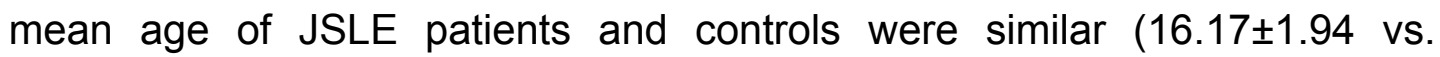
$16.13 \pm 2.16$ years, $p=0.92$ ). The cervicovaginal cytology was found to be similar in both groups, although sexual intercourses in the last month were less frequent in JSLE than controls ( $23 \%$ vs. $59.6 \%, p=0.0003)$. Only one patient $(2 \%)$ with JSLE versus two controls $(4 \%)$ had cervical dysplasia (LGSIL) and human papilomavirus $(p=1.0)$. Remarkably, inflammatory cervicovaginal cytology was observed in $21(60 \%)$ of patients with SLEDAI $\geq$ 4 and only $4(23 \%)$ of those with SLEDAI $<4(p=0.001)$. Likewise, a higher frequency of inflammatory changes were also observed in virgin JSLE $(57 \%$ vs. $8 \%, p=0.005)$. Of interest, Candida spp vaginitis was observed in 7 JSLE $(14 \%)$ versus none in controls $(p=0.012)$ and was associated to immunosuppressive drugs $(p=0.01)$ and high dose of prednisone $(p=0.002)$. Our findings supports the notion that female genital tract is a target organ in SLE since cervical inflammation is associated to disease activity independently of sexual activity.

Keywords: Juvenile systemic lupus erythematosus, cervicovaginal cytology, pap smears, vaginal infection, adolescent 


\section{INTRODUCTION}

Juvenile systemic lupus erythematosus (JSLE) is a multisystem autoimmune disease of unknown etiology affecting especially young females. ${ }^{1}$ The recently expanded understanding of the genetic, immunopathologic mechanisms and novel therapies of JSLE has resulted in improved 10-year survival rates that are now found to be over $80 \%$ in these patients. $^{2,3}$

As JSLE patients are living longer, clinical attention has begun to shift away to improve their quality of life. In this regard, upon becoming adolescents, female JSLE became sexually active with a consequent higher risk of sexually transmitted disease (STD), vaginitis and cervical dysplasia.

Of note, vaginitis is one of the most common infections in women in all age groups. ${ }^{4}$ Furthermore evidence suggests that vaginitis, particularly vulvovaginal candidiasis, is an even greater problem for patients that suffer from chronic illness and immunosuppression. ${ }^{4-6}$

Likewise, lupus itself may be associated with an enhanced risk of cervical dysplasia, since the described frequency in adult SLE ranging of $24 \%$ to $36 \%$ is much higher than that observed for the control group. ${ }^{7-11}$ In addition, human papillomatovirus (HPV) infection ${ }^{10}$ and immunosuppressive drugs particularly intravenous cyclophosphamide (IVCYC) and azathioprin ${ }^{12-}$ ${ }^{14}$ may also induce the development of abnormal Papanicolaou (Pap) smears in SLE patients.

In juvenile lupus however, there are no data regarding the frequency of vaginitis and cervical dysplasia, as well as its possible association with immunosupressive drugs, disease activity and damage. Therefore, the aim of this cross-sectional study was to evaluate cervicovaginal cytology in JSLE adolescents and compare to controls. 


\section{METHODS}

Patients and controls: Fifty-five consecutive female JSLE patients with age between 10-19 years old regularly followed at the Pediatric Rheumatology Unit or the Rheumatology Division, University of São Paulo, were selected for this study from 2004 to 2005. All patients fulfilled the American College of Rheumatology (ACR) SLE classification criteria. ${ }^{15}$ Inclusion criteria was presence of menarche and exclusion criteria were current pregnancy and diabetes mellitus. Three patients had parental refusal. After exclusion, the final group consisted of 52 JSLE patients. The control group consisted of 52 healthy female adolescents age-matched followed at the Adolescent Gynecology Unit of our University Hospital. The Local Ethical Committee approved this study and an informed consent was obtained from all participants and when necessary from their respective parents. The Brazilian socio-economic classes were classified according the Associação Brasileira dos Institutos de Pesquisa de Mercados. ${ }^{16}$

\section{Clinical evaluation, laboratory evaluation and treatment of JSLE} patients: An extensive clinical evaluation at entry by the same rheumatologist (MVF) was followed by a careful chart review including previous clinical, laboratory and therapeutic data. JSLE manifestations were defined as: cutaneous lesions (malar or discoid rash, oral ulcers, vasculitis or photosensitivity), articular involvement (arthralgia or nonerosive arthritis), neuropsychiatry disease (seizure, psychosis, depression, or peripheral neuropathy), renal involvement (proteinuria $\geq 0.5 \mathrm{~g} / 24 \mathrm{~h}$, presence of cellular casts, persistent hematuria $\geq 10$ red blood cells per high power field, or renal failure), cardiopulmonary disease (serositis, myocarditis, restrictive lung disease, and pulmonary hypertension), and hematologic abnormalities (hemolytic anemia, leukopenia with a white blood cell count $<4,000 / \mathrm{mm}^{3}$, lymphopenia $<1,500 / \mathrm{mm}^{3}$ on two or more occasions, and thrombocytopenia with platelet count $<100,000 / \mathrm{mm}^{3}$ in the absence of drugs or infection). 
SLE disease activity and cumulative damage at the time of study entry were measured in all patients, using the SLE Disease Activity Index $\left(\right.$ SLEDAI ${ }^{17}$ and the Systemic Lupus International Collaborating Clinics/ACR (SLICC/ACR) Damage Index. ${ }^{18}$ Data concerning the current dosage of prednisone, methotrexate, azathioprine, IVCYC, cyclosporine and mycofenolate mofetil were determined.

Gynecologic evaluation: A systematic clinical examination of the genitalia was performed by the same expert gynecologist at study entry and includes evaluation of vulva, hymen, vagina and cervix, secondary sexual characteristics according to Marshall and Tanner's pattern of pubertal changes ${ }^{19}$ and collection of specimens for a Pap test in the follicular phase of menstrual cycle.

Patients were evaluated according to: age at menarche; age of the first sexual relation; frequency of sexual intercourse (in the last month), number of sexual partners (in the last year); vaginitis (candidiasis, bacterial vaginosis, Trichomonas vaginallis, HPV, etc) and contraception.

Cervicovaginal cytology: The Pap smears were collected in virgin adolescents with Cytobrush $\AA$, the brush was inserted in the band of vaginal ostium and gently rotated $90^{\circ}$ to $180^{\circ}$, and immediately rolled over the outer third of the slide. In sexually active adolescents, the cervicovaginal cytology was collected with Cytobrush $\circledast$ and spatula Ayre, after insertion of the speculum. The cervix was visualized and the spatula Ayre was inserted in the ostium and rotated $360^{\circ}$ inder gently pressure, after inserted the Cytobrush ${ }^{\circledR}$ for two thirds in the endocervical canal and rotated $90^{\circ}$ to $180^{\circ}$. The material of the Cytobrush ${ }^{\circledR}$ was rolled over the outer third of the slide and the material on the spatula Ayre was spread in a thin layer in one movement over the middle third of the slide. After the fixation by immersion in $95 \%$ ethanol, the specimens were immediately transported to the laboratory. ${ }^{20}$

All Pap smears were evaluated by the same cytopathologist blinded to gynecology examination in our University Hospital. They were performed 
according to the Bethesda Classification System in 5 patterns with presence or absence of HPV infection: normal (benign cellular changes), inflammatory changes, atypical squamous cells of uncertain significance (ASCUS), high- or low-grade squamous intraepithelial lesions (HGSIL or LGSIL), and carcinoma in situ. The identification of the organisms (Trichomonas vaginalis, Candida spp, bacterial vaginosis, Actinomyces spp, herpes simplex virus) was also evaluated. $^{21}$

Statistical analysis: Results were presented as the mean \pm standard deviation (SD) or median for continuous and number (\%) for categorical variables. Data were compared by $t$ test in continuous variables to evaluate differences among JSLE and control group, and among JSLE subgroups. For categorical variables differences were assessed by chi-square test or Fisher's exact test. In all the statistical tests the level of significance was set at $5 \%(p<0.05)$. 


\section{RESULTS}

Demographic features: The distribution of demographic features revealed that JSLE patients and controls were comparable regarding mean current age (16.17 \pm 1.94 vs. $16.13 \pm 2.16$ years, $p=0.92)$, Brazilian socioeconomic class $(p=0.33)$ and number of school years $(9.51 \pm 1.78$ vs. $9.7 \pm$ 1.62 years, $p=0.57)$.

Gynecologic evaluation: The secondary sexual characteristics according to Marshall and Tanner's pattern of pubertal changes, ${ }^{19}$ were similar in both groups with regard to the predominance of M4P4 (48\% vs. $46 \%, p=1.0)$. Of note, the mean age of menarche of JSLE patients (12.82 \pm 1.62 years, range 10 to 17$)$ was higher than control group (11.54 \pm 1.45 years, range 9 to $14, p=0.00004)$. On the other hand, the frequency of sexually active patients was lower in JSLE patients than controls (23\% vs. $60 \%, p=0.0003)$. In contrast, no difference was observed concerning the mean age of the first sexual intercourse $(15.33 \pm 1.72$ vs. $14.87 \pm 1.99$ years, $p=0.48)$, the mean number of sexual intercourses in the last month (4.33 \pm 4.73 vs. $5.45 \pm 4.02$ days, $p=0.44$ ) and the median number of the partners in the last year [1 (1-5) vs. 1 (1-10), $p=1.0]$ in JSLE and controls sexually active. Furthermore, a similar frequency of contraception ( $83 \%$ vs. $90 \%, p=0.61)$ and condom use in partners of those sexually active (92\% vs. $70 \%, p=0.65)$ was found in JSLE and control group.

Cervicovaginal cytology: Normal Pap smears were observed in $50 \%$ of both groups $(p=1.15)$, inflammatory changes in $48 \%$ of JSLE and $46 \%$ of controls $(p=1.0)$. LGSIL with presence of HPV infection were observed in only one patient with JSLE (2\%) versus two controls $(4 \%)(p=1.0)($ Table 1$)$, spite of the fact that $27(52 \%)$ JSLE patients were under of one or more immunosuppressive drugs (azathioprine in 17, IVCYC in 15, methotrexate in 11, cyclosporine in 2 and mycofenolate mofetil in 1). On the other hand, among the 12 JSLE and 31 controls sexually active, no differences were 
observed regarding frequency of normal, inflammatory and LGSIL comparing sexually actives JSLE patients and sexually actives controls (25\% vs. $29 \%$, $p=1.0 ; 67 \%$ vs. $65 \%, p=1.0 ; 8 \%$ vs. $6 \%, p=1.0 ;$ respectively).

Interestingly, inflammatory changes in cervicovaginal cytology were significantly associated with disease activity in JSLE with and without sexual activity. In fact, Pap smears with inflammatory changes was observed in $60 \%$ of patients with SLEDAI $\geq 4$ versus $23.5 \%$ of patients with SLEDAI $<4$ $(p=0.001)$. Reinforcing this finding, only $37 \%$ of patients with SLEDAI $\geq 4$ had normal cervicovaginal cytology whereas $77 \%$ of those with SLEDAI $<4$ had normal pattern $(p=0.001)$ (Table 2$)$. Likewise, the specific analysis of the virgin JSLE patients revealed that inflammatory changes was also observed more frequently in patients with SLEDAI $>4$ than in those with SLEDAI $<4$ (57\% vs.8\%, respectively, $p=0.005$ ) (Table 3 ). In contrast, no statistical association was observed in relation to SLICC/ACR-DI $\geq 1$ or $<1$ and inflammatory changes ( $48 \%$ vs. $50 \%, p=1.0)$.

Organisms: Remarkably, 7 (14\%) of JSLE patients evidenced Candida spp in Pap smears compared to $0 \%$ of controls $(p=0.01)$ (Table 1). All of them had inflammatory changes in Pap smears and five were virgin. Likewise, in sexually active individuals, a higher frequency of Candida spp was observed in lupus compared to controls ( $17 \%$ vs. $0 \%$, respectively), although this difference did not reach statistical significance $(p=0.07)$. Only 2 patients had candidiasis with vaginal discomfort and leucorrhea, and 5 had only leucorrhea. All patients with Candida spp were treated with oral and topic fluconazole therapy for 7 days and showed symptomatic improvement. Of note, JSLE patients with Candida spp were under higher mean current dose of prednisone compared to those without this vaginal organism (20.5 \pm 5 vs. $10.2 \pm 8 \mathrm{mg}, p=0.002$ ). Likewise, the percentage of the immunosuppressive drugs use (azathioprine and/or IVCYC) was higher in patients with Candida spp (100\% vs. 44\%, p=0.01) (Table 4). In addition, frequencies of clinical findings were evenly observed in JSLE with or without Candida spp: articular ( $86 \%$ vs. $89 \%, p=1.0)$, hematological abnormalities 
( $86 \%$ vs. $93 \%, p=0.44)$, cutaneous ( $100 \%$ vs. $96 \%, p=1.0)$, renal $(100 \%$ vs. $82 \%, p=0.57)$, cardiopulmonary ( $57 \%$ vs. $42 \%, p=0.68)$, neuropsychiatry ( $29 \%$ vs. $22 \%, p=0.65)$. The mean of SLEDAI score $(5.4 \pm 4.2$ vs. $5.5 \pm 4.4$, $p=0.95)$ and percentage of SLICC-ACR/DI score $\geq 1$ (43\% vs. $16 \%, p=0.3)$ were comparable in JSLE patients with the presence or absence of Candida spp in Pap smears (Table 4).

Condylomata acuminata by HPV infection was identified in vulva, vagina and/or cervix by colposcopy in two sexually actives controls (6.4\%) and none in JSLE patients $(p=1.0)$. These patients had LGSIL and were treated with loop electrosurgical excisional procedure.

Bacterial vaginosis by Gardnerella vaginallis were observed in 3 (10\%) of sexually actives controls compared to none sexually actives JSLE patients $(p=0.54)$. These patients presented a malodorous vaginal discharge that was tipically thin and dull gray in color. The treatment of bacterial vaginosis consisted of metronidazol topical.

Of note, Trichomonas vaginalis, Actinomyces species and cellular changes consisted with herpes simplex virus were not found in Pap smears of JSLE patients and controls. 


\section{DISCUSSION}

This is the first study that specifically addressed cervicovaginal cytology in adolescents JSLE and clearly demonstrates a high frequency of inflammatory changes in Pap smears and fungal vaginitis.

Vaginitis is a major problem for immunocompromised states such as diabetes mellitus, HIV and autoimmune diseases. ${ }^{6}$ In fact, pathological situations in which the vaginal $\mathrm{pH}$ rises may result in suppression of the growth of lactobacilli and overgrowth of potentially pathogenic bacteria or yeasts, and leads to vaginitis. ${ }^{4}$

In health women, the most common vaginal infections are Candidal vaginitis, bacterial vaginosis and Trichomonas vaginalis. ${ }^{4}$ In contrast, no data is available in lupus regard vaginitis, in spite of several studies demonstrating that infection is a major cause of morbidity and mortality in lupus patients. ${ }^{22}$

In the present study, Candida spp was identified as the major pathogenic organism of vaginitis in JSLE. Excessive epithelial adhesion and colonization of the Candida spp are related to the adherence capability of the fungus as well as the poor innate immunity in the compromised host. Furthermore, vaginal candidiasis is caused by defective or dysfunctional CD4+ T helper 1-type cell-mediated immune reactivity. Moreover, peripheral blood lymphocytes from patients who are receiving high doses of prednisone have been shown to have markedly depressed in vivo lymphocyte transformation to antigenic stimulation by Candida spp. ${ }^{23}$ In this regard, JSLE patients receiving immunosupressive drugs should be considered candidates for fungal infections. In fact, all patients with this opportunistic infection evaluated herein were under immunosupressive therapy and a higher dose of prednisone.

Importantly, the female genital tract should be considered as a primary site of candidiasis, with a chance of systemic dissemination since one previous study have demonstrated that Candida albicans was the most frequent opportunistic organism identified in fatal infection in adult SLE. ${ }^{24}$ 
In our study we have not performed culture to identify the yeast species because it is largely accepted that it is not necessary in view of the fact that most Candida spp will be sensitive to topical fluconazole, ${ }^{25}$ clotrimazole or miconazole ${ }^{4}$ as also observed herein.

Of interest, cervical dysplasia, particularly low-grade squamous intraepithelial lesions by the Bethesda Classification System, was rarely observed in JSLE and controls. This finding is consistent with others studies with healthy adolescents that showed cervical dysplasia in $1.9 \%$ to $37 \%$ and LGSIL in $3 \%$ to $24.5 \%{ }^{26-30}$ The most likely explanation for our finding is the lower frequency of sexual activity possibly related to delayed menarche in JSLE compared to controls. Indeed, we have previously showed that adolescents with JSLE presented a late occurrence of their first period (13.5 \pm 1.4 years) in comparison with 2578 normal Brazilian adolescents (12.5 \pm 1.3$).{ }^{31}$

Of note, previous studies in women with SLE have demonstrated an increased prevalence of atypical cervical smears with LGSIL ranging of $12 \%$ to $36 \%^{8-10,12}$ and were associated to immunosuppressive drugs use, particularly IVCYC and azathioprine..$^{8,12-14}$ The low incidence of cervical dysplasia in our patients in spite of high frequency and long-term immunosuppressive use, suggest that sexual activity is probably the main contributing factor for this Pap smears abnormality.

Actually, the incidence of cervical abnormalities among adolescents is related primarily to increasing rates of sexual activity, promiscuity and HPV infection. ${ }^{28,32-34} \mathrm{~A}$ possible explanation for this association is inherent vulnerability of the "immature" cervix that is covered predominantly columnar and metaplastic epithelium and reflects rapid replication and differentiation, this epithelial type is perfect host for HPV replication. ${ }^{33}$ The frequent use of condom use by JSLE and controls partners in the present study probably have contributed to the low incidence of cervical and vulvovaginal HPV infection as recently demonstrated for young sexually active women. ${ }^{35}$

On the other hand, SLE itself may predispose to cervical inflammation since this alteration in cervical cytology was associated to higher SLEDAI score. Remarkably, this finding was also true for virgin JSLE patients, who 
are not exposed to sexually transmited genital infection, reinforcing the role of disease activity in cervical inflammatory alterations. Moreover, since the later were not secondary to local infection in the majority of the patients studied herein we hypothesize that female genital tract is indeed a target organ in lupus.

\section{REFERENCES}

1. Benseler SM, Silverman ED. Systemic lupus erythematosus. Pediatr Clin N Am 2005; 52: 443-467.

2. Arkachaisri T, Lehman TJ. Systemic lupus erythematosus and related disorders of childhood. Curr Opin Rheumatol 1999; 11: 384-392.

3. Stichweh D, Arce E, Pascual V. Update on pediatric systemic lupus erythematosus. Curr Opin Rheumatol 2004; 16: 577-587.

4. Aagaard-Tillery KM, Holmgren CM, Scott JR. Gynecologic Problems in Women with Autoimmune Diseases. In: Lockshin M, Branch DW. Handbook of Systemic Autoimune Diseases, Volume 4. Reproductive and Hormonal Aspects of Systemic Autoimmune Diseases. 1st edition. Elsevier, 2006, pp 141-160.

5. Fidel PL Jr. Immunity in vaginal candidiasis. Curr Opin Infect Dis 2005; 18 : 107-111.

6. Fidel PL Jr, Sobel JD. Immunopathogenesis of recurrent vulvovaginal candidiasis. Clin Microbiol Rev 1996; 9: 335-348.

7. Blumenfeld Z, Lorber M, Yoffe N, Scharf Y. Systemic lupus erythematosus: predisposition for uterine cervical dysplasia. Lupus 1994; 3: 59-61.

8. Nyberg G, Eriksson O, Westberg NG. Increased incidence of cervical atypia in women with systemic lupus erythematosus treated with chemotherapy. Arthritis Rheum 1981; 24: 648-650.

9. Dhar JP, Kmak D, Bhan R, Pishorodi L, Ager J, Sokol RJ. Abnormal cervicovaginal cytology in women with lupus: a retrospective cohort study. Gynecol Oncol 2001; 82: 4-6. 
10. Tam LS, Chan AY, Chan PK, Chang AR, Li EK. Increased prevalence of squamous intraepithelial lesions in systemic lupus erythematosus: association with human papillomavirus infection. Arthritis Rheum 2004; 50: 3619-3625.

11. Dhar JP, Essenmacher L, Ager J, Sokol RJ. Ominous cervical cytopathology in women with lupus. Int J Gynaecol Obstet. 2005; 89: 295-296.

12. Bateman H, Yazici Y, Leff L, Peterson M, Paget SA. Increased cervical dysplasia in intravenous cyclophosphamide treated patients with SLE: a preliminary study. Lupus 2000; 9: 542-4.

13. Ognenovski VM, Marder W, Somers EC et al. Increased incidence of cervical intraepithelial neoplasia in women with Systemic Lupus Erythematosus treated with intravenous cyclophosphamide. J Rheumatol 2004; 31: 1763-1737.

14. Bernatsky S, Ramsey-Goldman R, Gordon C, et al. Factors associated with abnormal Pap results in systemic lupus erythematosus. Rheumatology 2004; 43: 1386-1389.

15. Hochberg MC. Updating the American College of Rheumatology revised criteria for the classification of systemic lupus erhytematosus. Arthrits Rheum 1997; 40: 1725.

16. Almeida PM, Wickerrhauser $H$. Critério de classe econômica da Associação Brasileira de Anunciantes (ABA) e Associação Brasileira dos Institutos de Pesquisa de Mercado (ABIPEME) 1991, pp 1-29.

17. Brunner HI, Silverman ED, To T, Bombardier C, Feldman BM. Sensitivity of the Systemic Lupus Erhytematosus Disease Activity Index, British Isles Lupus Assessement Group Index, and Systemic Lupus Activity Measure in the evaluation of clinical change in childhood-onset systemic lupus erhytematosus. Arthrits Rheum 1999; 42: 1354-1360.

18. Brunner HI, Silverman ED, To T, Bombardier C, Feldman BM. Risk factors for damage in childhood-onset systemic lupus erythematosus: cumulative disease activity and medication use predict disease damage. Arthritis Rheum 2002; 46: 436-444. 
19. Marshall JC, Tanner JM. Variations in patterns of pubertal changes in boys and girls. Arch Dis Child 1970; 45: 13-23.

20. Fokke HE, Salvatore CM, Schipper MEI, Bleker OP. A randomized trial of three methods of obtaining Papanicolaou semars. Eu J Obstet Gynecol Reprod Bio 1993; 48: 103-106.

21. Solomon D, Davey D, Kurman R et al. The 2001 Bethesda System: terminology for reporting results of cervical cytology. JAMA 2002; 287: 2114-9.

22. Greenberg SB. Infections in the immunocompromised rheumatologic patient. Crit Care Clin 2002; 18: 931-956.

23. Folb PI, Trounce JR. Immunological aspects of candida infection complicating steroid and immunosuppressive drug therapy. Lancet 1970; 2: $1112-1114$.

24. Hellmann DB, Petri M, Whiting-O'Keefe Q. Fatal infections in systemic lupus erythematosus: the role of opportunistic organisms. Medicine (Baltimore) 1987; 66: 341-348.

25. Goswami D, Goswami R, Banerjee U et al. Pattern of Candida species isolated from patients with diabetes mellitus and vulvovaginal candidiasis and their response to single dose oral fluconazole therapy. J Infect 2006; 52: 111-117.

26. Tarkowski TA, Koumans EH, Sawyer M et al. Epidemiology of human papillomavirus infection and abnormal cytologic test results in an urban adolescent population. J Infect Dis 2004; 189: 46-50.

27. Wright JD, Davila RM, Pinto KR et al. Cervical dysplasia in adolescents. Obstet Gynecol 2005; 106: 115-120.

28. Utagawa ML, Pereira SM, Cavaliere MJ, Maeda MY, Shih LW, Shirata NK. Cervical intraepithelial neoplasia in adolescents: study of cytological findings between 1987 and 1995 in Sao Paulo State-Brazil. Arch Gynecol Obstet 1998; 262: 59-64.

29. Prussia PR, Gay GH, Bruce A. Analysis of cervico-vaginal (Papanicolaou) smears, in girls 18 years and under. West Indian Med J 2002; 51: 37-39. 
30. Simsir A, Brooks S, Cochran L, Bourquin P, loffe OB. Cervicovaginal smear abnormalities in sexually active adolescents. Implications for management. Acta Cytol 2002; 46: 271-276.

31. Silva CA, Leal MM, Leone $\mathrm{C}$ et al. Gonadal function in adolescents and young women with juvenile systemic lupus erythematosus. Lupus 2002; 11: $419-425$.

32. Mount SL, Papillo JL. A study of 10,296 pediatric and adolescent Papanicolaou smear diagnoses in northern New England. Pediatrics 1999; 103: 539-545.

33. Moscicki AB. Human Papilloma Virus, Papanicolaou Smears, and the College Female. Pediatr Clin N Am 2005; 52: 167-177.

34. Hillard PJA. Pediatric and adolescent gynecology. In: Scott JR, Saia PJ, Hammond CB, Spellacy WN. Danforth's Obstetrics \& Gynecology, 9th edition. Lippincott Williams \& Wilkins, 1999, pp 529-540.

35. Winer RL, Hughes JP, Feng Q, et al. Condom use and the risk of genital human papillomavirus infection in young women. N Engl J Med 2006; 354: 2645-2654. 
Table 1 - Cervicovaginal cytology of JSLE patients and controls.

\begin{tabular}{lccc}
\hline Cervicovaginal cytology , $\mathbf{n}(\%)$ & $\begin{array}{c}\text { JSLE } \\
(\mathbf{n = 5 2})\end{array}$ & $\begin{array}{c}\text { Controls } \\
(\mathbf{n}=52)\end{array}$ & $\mathbf{P}$ \\
\hline Cytopathology & & & \\
$\quad$ Normal & $26(50)$ & $26(50)$ & 1.15 \\
Inflammatory & $25(48)$ & $24(46)$ & 1.0 \\
LGSIL & $1(2)$ & $2(4)$ & 1.0 \\
Organisms & $0(0)$ & $4(8)$ & 0.11 \\
Bacterial vaginosis & $7(14)$ & $0(0)$ & 0.01 \\
Candida spp & & \\
\hline
\end{tabular}

JSLE - Juvenile Systemic Lupus Erythemathosus, LGSIL - low grade squamous intraepithelial lesions. 
Table 2 - Characteristics of JSLE cervicovaginal cytology according to disease activity.

\begin{tabular}{|c|c|c|c|}
\hline Cervicovaginal cytology, $\mathbf{n}(\%)$ & $\begin{array}{c}\text { SLEDAI < } 4 \\
(n=17)\end{array}$ & $\begin{array}{c}\text { SLEDAI } \geq 4 \\
(n=35)\end{array}$ & $\mathbf{p}$ \\
\hline Normal & $13(77)$ & $13(37)$ & 0.001 \\
\hline Inflammatory & $4(23)$ & $21(60)$ & 0.001 \\
\hline LGSIL & $0(0)$ & $1(3)$ & 1.0 \\
\hline
\end{tabular}


Table 3 - Characteristics of JSLE cervicovaginal cytology in virgin adolescents according to disease activity.

\begin{tabular}{lccc}
\hline Cervicovaginal cytology, $\mathbf{n}(\mathbf{\%})$ & $\begin{array}{c}\text { SLEDAl }<\mathbf{4} \\
(\mathbf{n = 1 2})\end{array}$ & $\begin{array}{c}\text { SLEDAI } \geq \mathbf{4} \\
(\mathbf{n = 2 8 )}\end{array}$ & $\mathbf{p}$ \\
\hline Normal & $11(92)$ & $12(53)$ & 0.005 \\
Inflammatory & $1(8)$ & $16(57)$ & 0.005 \\
\hline JSLE - Juvenile Systemic & Lupus & Erythemathosus, SLEDAI & - \\
Systemic Lupus Erythematosus Disease Activity Index.
\end{tabular}


Table 4 - Clinical features, activity, damage and treatment of JSLE patients with and without vaginal infection by Candida spp.

\begin{tabular}{|c|c|c|c|}
\hline Variables & $\begin{array}{c}\text { With } \\
\text { Candida spp } \\
(\mathrm{n}=7)\end{array}$ & $\begin{array}{c}\text { Without } \\
\text { Candida spp } \\
(\mathrm{n}=45)\end{array}$ & $\mathbf{p}$ \\
\hline \multicolumn{4}{|l|}{ Clinical features } \\
\hline Cutaneous n (\%) & $7(100)$ & $43(96)$ & 1.0 \\
\hline Articular, n (\%) & $6(86)$ & $40(89)$ & 1.0 \\
\hline Renal, n (\%) & $7(100)$ & $37(82)$ & 0.57 \\
\hline Cardiopulmonary, n (\%) & $4(57)$ & $19(42)$ & 0.68 \\
\hline Neuropsychiatry, n (\%) & $2(29)$ & $10(22)$ & 0.65 \\
\hline Hematological abnormalities, n (\%) & $6(86)$ & $42(93)$ & 0.44 \\
\hline \multicolumn{4}{|l|}{ JSLE activity and damage } \\
\hline SLEDAI, mean \pm SD & $5.4 \pm 4.2$ & $5.5 \pm 4.4$ & 0.95 \\
\hline SLICC/ACR-DI $\geq 1, \mathrm{n}(\%)$ & $3(4)$ & $7(16)$ & 0.3 \\
\hline \multicolumn{4}{|l|}{ Treatment } \\
\hline Current dose of $\mathrm{Pd}(\mathrm{mg})$, mean $\pm \mathrm{SD}$ & $20.5 \pm 5$ & $10.2 \pm 8$ & 0.002 \\
\hline Current use of IS, n (\%) & $7(100)$ & $20(44)$ & 0.01 \\
\hline
\end{tabular}

JSLE - Juvenile Systemic Lupus Erythemathosus, IS - immunosuppressive drugs, Pd - prednisone, SLEDAI - Systemic Lupus Erythemathosus Disease Activity Index, SLICC/ACR-DI - Systemic Lupus International Collaborating Clinics/American College of Rheumatology-Damage Index, SD - standard deviation. 
8. REFERÊNCIAS 
1. Benseler SM, Silverman ED. Systemic lupus erythematosus. Pediatr Clin N Am 2005; 52: 443-467.

2. Arkachaisri T, Lehman TJ. Systemic lupus erythematosus and related disorders of childhood. Curr Opin Rheumatol 1999; 11: 384-392.

3. Stichweh D, Arce E, Pascual V. Update on pediatric systemic lupus erythematosus. Curr Opin Rheumatol 2004; 16: 577-587.

4. Aagaard-Tillery KM, Holmgren CM, Scott JR. Gynecologic Problems in Women with Autoimmune Diseases. In: Lockshin M, Branch DW. Handbook of Systemic Autoimune Diseases, Volume 4. Reproductive and Hormonal Aspects of Systemic Autoimmune Diseases. 1st edition. Elsevier, 2006, pp 141-160.

5. Fidel PL Jr. Immunity in vaginal candidiasis. Curr Opin Infect Dis 2005; 18 : 107-111.

6. Fidel PL Jr, Sobel JD. Immunopathogenesis of recurrent vulvovaginal candidiasis. Clin Microbiol Rev 1996; 9: 335-348.

7. Blumenfeld Z, Lorber M, Yoffe N, Scharf Y. Systemic lupus erythematosus: predisposition for uterine cervical dysplasia. Lupus 1994; 3: 59-61.

8. Nyberg G, Eriksson O, Westberg NG. Increased incidence of cervical atypia in women with systemic lupus erythematosus treated with chemotherapy. Arthritis Rheum 1981; 24: 648-650. 
9. Dhar JP, Kmak D, Bhan R, Pishorodi L, Ager J, Sokol RJ. Abnormal cervicovaginal cytology in women with lupus: a retrospective cohort study. Gynecol Oncol 2001; 82: 4-6.

10. Tam LS, Chan AY, Chan PK, Chang AR, Li EK. Increased prevalence of squamous intraepithelial lesions in systemic lupus erythematosus: association with human papillomavirus infection. Arthritis Rheum 2004; 50: 3619-3625.

11. Dhar JP, Essenmacher L, Ager J, Sokol RJ. Ominous cervical cytopathology in women with lupus. Int J Gynaecol Obstet. 2005; 89: 295-296.

12. Bateman H, Yazici $Y$, Leff L, Peterson M, Paget SA. Increased cervical dysplasia in intravenous cyclophosphamide treated patients with SLE: a preliminary study. Lupus 2000; 9:542-4.

13. Ognenovski VM, Marder W, Somers EC, Johnston CM, Farrehi JG, Selvaggi SM, McCune WJ. Increased incidence of cervical intraepithelial neoplasia in women with Systemic Lupus Erythematosus treated with intravenous cyclophosphamide. J Rheumatol 2004; 31: 1763-1737.

14. Bernatsky S, Ramsey-Goldman R, Gordon C, Joseph L, Boivin JF, Rajan $\mathrm{R}$, Allen $\mathrm{A}$, Moore $\mathrm{AD}$, Leung $\mathrm{MH}$, Clarke $\mathrm{A}$. Factors associated with abnormal Pap results in systemic lupus erythematosus. Rheumatology 2004; 43: 1386-1389.

15. Hochberg MC. Updating the American College of Rheumatology revised criteria for the classification of systemic lupus erhytematosus. Arthrits Rheum 1997; 40: 1725.

16. Almeida PM, Wickerrhauser $H$. Critério de classe econômica da Associação Brasileira de Anunciantes (ABA) e Associação Brasileira dos Institutos de Pesquisa de Mercado (ABIPEME) 1991, pp 1-29. 
17. Brunner HI, Silverman ED, To T, Bombardier C, Feldman BM. Sensitivity of the Systemic Lupus Erhytematosus Disease Activity Index, British Isles Lupus Assessement Group Index, and Systemic Lupus Activity Measure in the evaluation of clinical change in childhood-onset systemic lupus erhytematosus. Arthrits Rheum 1999; 42: 1354-1360.

18. Brunner HI, Silverman ED, To T, Bombardier C, Feldman BM. Risk factors for damage in childhood-onset systemic lupus erythematosus: cumulative disease activity and medication use predict disease damage. Arthritis Rheum 2002; 46: 436-444.

19. Marshall JC, Tanner JM. Variations in patterns of pubertal changes in boys and girls. Arch Dis Child 1970; 45: 13-23.

20. Fokke HE, Salvatore CM, Schipper MEl, Bleker OP. A randomized trial of three methods of obtaining Papanicolaou semars. Eu J Obstet Gynecol Reprod Bio 1993; 48: 103-106.

21. Solomon D, Davey D, Kurman R, Moriarty A, O'Connor D, Prey M, Raab S, Sherman M, Wilbur D, Wright T, Young N. The 2001 Bethesda System: terminology for reporting results of cervical cytology. JAMA 2002; 287:2114-9.

22. Greenberg SB. Infections in the immunocompromised rheumatologic patient. Crit Care Clin 2002; 18: 931-956.

23. Folb PI, Trounce JR. Immunological aspects of candida infection complicating steroid and immunosuppressive drug therapy. Lancet 1970; 2: $1112-1114$.

24. Hellmann DB, Petri M, Whiting-O'Keefe Q. Fatal infections in systemic lupus erythematosus: the role of opportunistic organisms. Medicine (Baltimore) 1987; 66: 341-348. 
25. Goswami D, Goswami R, Banerjee U, Dadhwal V, Miglani S, Lattif AA, Kochupillai N. Pattern of Candida species isolated from patients with diabetes mellitus and vulvovaginal candidiasis and their response to single dose oral fluconazole therapy. J Infect 2006; 52: 111-117.

26. Tarkowski TA, Koumans EH, Sawyer M, Pierce A, Black CM, Papp JR, Markowitz L, Unger ER. Epidemiology of human papillomavirus infection and abnormal cytologic test results in an urban adolescent population. $J$ Infect Dis 2004; 189: 46-50.

27. Wright JD, Davila RM, Pinto KR, Merritt DF, Gibb RK, Rader JS, Mutch DG, Gao F, Powell MA. Cervical dysplasia in adolescents. Obstet Gynecol 2005; 106: 115-120.

28. Utagawa ML, Pereira SM, Cavaliere MJ, Maeda MY, Shih LW, Shirata NK. Cervical intraepithelial neoplasia in adolescents: study of cytological findings between 1987 and 1995 in Sao Paulo State-Brazil. Arch Gynecol Obstet 1998; 262: 59-64.

29. Prussia PR, Gay GH, Bruce A. Analysis of cervico-vaginal (Papanicolaou) smears, in girls 18 years and under. West Indian Med J 2002; 51: 37-39.

30. Simsir A, Brooks S, Cochran L, Bourquin P, loffe OB. Cervicovaginal smear abnormalities in sexually active adolescents. Implications for management. Acta Cytol 2002; 46: 271-276.

31. Silva CA, Leal MM, Leone C, Simone VP, Takiuti AD, Saito MI, Kiss MHB. Gonadal function in adolescents and young women with juvenile systemic lupus erythematosus. Lupus 2002; 11: 419-425.

32. Mount SL, Papillo JL. A study of 10,296 pediatric and adolescent Papanicolaou smear diagnoses in northern New England. Pediatrics 1999; 103: 539-545. 
33. Moscicki AB. Human Papilloma Virus, Papanicolaou Smears, and the College Female. Pediatr Clin N Am 2005; 52: 167-177.

34. Hillard PJA. Pediatric and adolescent gynecology. In: Scott JR, Saia PJ, Hammond CB, Spellacy WN. Danforth's Obstetrics \& Gynecology, 9th edition. Lippincott Williams \& Wilkins, 1999, pp 529-540.

35. Winer RL, Hughes JP, Feng Q, Feng Q, O'Reilly S, Kiviat NB, Holmes KK, Koutsky LA. Condom use and the risk of genital human papillomavirus infection in young women. N Engl J Med 2006; 354: 2645-265. 